\title{
Mode of action of pertuzumab in combination with trastuzumab plus docetaxel therapy in a HER2-positive breast cancer xenograft model
}

\author{
YORIKO YAMASHITA-KASHIMA, SEI SHU, KEIGO YOROZU, YOICHIRO MORIYA and NAOKI HARADA \\ Product Research Department, Kamakura Research Laboratories, Chugai Pharmaceutical Co., Ltd., \\ Kamakura, Kanagawa 247-8530, Japan
}

Received December 9, 2015; Accepted June 23, 2016

DOI: $10.3892 / \mathrm{ol} .2017 .6679$

\begin{abstract}
In a Phase III trial for HER2-positive breast cancer (the CLEOPATRA study), the triple-drug combination arm of pertuzumab plus trastuzumab plus docetaxel showed significantly longer progression-free survival and overall survival than did the trastuzumab plus docetaxel arm. In this study, we investigated the mechanism of action of the triple-drug combination therapy in vivo. For this purpose, we established a mouse xenograft model using KPL-4, a HER2-positive human breast cancer cell line, in which the triple-drug combination treatment dramatically induced tumor regression compared with double-drug combinations (trastuzumab plus docetaxel, pertuzumab plus docetaxel, or pertuzumab plus trastuzumab). Four days after the triple-drug treatment was started, strong reduction in the phosphorylation of HER2, epidermal growth factor receptor (EGFR), HER3, extracellular signal-regulated kinase (ERK), and AKT in tumor tissues was seen, despite only weak suppression of phosphorylation seen with the single- or double-drug treatments. Histopathological analysis and flow cytometric analysis showed that the triple-drug treatment enhanced apoptosis after mitotic arrest induced by docetaxel. Furthermore, infiltration of mononuclear cells around the tumor cells was strongly induced by the triple-drug combination treatment. These results suggested that the mechanism underlying the synergistic efficacy of the triple-drug combination was attributable, at least in part, to the docetaxel-mediated apoptosis being promoted by enhanced inhibition of HER2-HER3-AKT signaling as well to the intratumor infiltration of mononuclear cells induced by anti-HER 2 antibodies being enhanced by docetaxel.
\end{abstract}

Correspondence to: DrNaoki Harada,Product Research Department, Kamakura Research Laboratories, Chugai Pharmaceutical Co., Ltd., 200 Kajiwara, Kamakura, Kanagawa 247-8530, Japan

E-mail: haradanok@chugai-pharm.co.jp

Key words: trastuzumab, pertuzumab, docetaxel, HER2, breast cancer

\section{Introduction}

The human epidermal growth factor receptor 2 (HER2, ErbB2/neu), a member of the ErbB/HER family proteins, is overexpressed in approximately $20 \%$ of human breast cancers and is positively associated with the aggressiveness of the disease and with poor prognosis (1-4). Therefore, HER2-targeted therapy is considered a very rational strategy for HER2-overexpressing breast cancer. Trastuzumab, the first humanized anti-HER2 monoclonal antibody, binds to domain IV of the HER2 extracellular domain (ECD) and inhibits ligand-independent HER2/HER3 signaling and HER2 shedding $(5,6)$. Trastuzumab also has the ability to trigger antibody-dependent cell-mediated cytotoxicity (ADCC) by binding to the Fc $\gamma$ receptor of immune cells such as natural killer cells through its Fc region $(7,8)$. Trastuzumab has been approved for the treatment of both early and metastatic HER2-overexpressing breast cancer. Although trastuzumab improves the survival of patients with advanced HER2-overexpressing cancer $(9,10)$, most patients eventually experience progressive disease. Therefore, a new treatment modality including trastuzumab was needed for advanced HER2-overexpressing cancer.

Pertuzumab is a humanized anti-HER2 monoclonal antibody that binds to a distinct epitope of HER2 (domain II) (11). Because domain II of HER2 is a region necessary for dimerization with other HER family receptors and signaling, pertuzumab inhibits ligand-induced dimerization and its downstream signaling $(11,12)$. In previous preclinical studies, we found that pertuzumab and trastuzumab bind to HER2 without competing with each other (13), and we and others have reported that the combination of pertuzumab plus trastuzumab exerts enhanced antitumor activity as compared to single-agent treatment $(13,14)$. In a Phase III clinical trial in patients with HER2-positive metastatic breast cancer (the CLEOPATRA study) it was demonstrated that the triple-drug combination of pertuzumab plus trastuzumab plus docetaxel, as compared to the combination of trastuzumab plus docetaxel, significantly improved progression-free survival and overall survival $(15,16)$. On the basis of that result, pertuzumab was firstly approved for HER2-positive metastatic breast cancer in combination with trastuzumab and chemotherapy. 
Nowadays, the triple-drug combination of pertuzumab plus trastuzumab plus docetaxel is becoming a first-line therapy for HER2-positive metastatic breast cancer. It is of enormous clinical importance, therefore, to give further thought to how the combination damages tumor cells and alters the tumor microenvironment. Preclinical studies using mouse xenograft models are a simple and effective way to investigate these questions. The aim of the present study was to assess the antitumor effect of the triple-drug combination from the aspect of cancer cell death and host immune cell response by using a human breast cancer xenografted mouse model.

\section{Materials and methods}

Test agents. Trastuzumab and pertuzumab were provided by $\mathrm{F}$. Hoffmann-La Roche (Basel, Switzerland) as a fine powder and a liquid, respectively. Trastuzumab was dissolved in distilled water. The two antibodies were then diluted with saline for the in vivo experiments and culture medium for the in vitro experiments. Human immunoglobulin $\mathrm{G}(\mathrm{HuIgG})$ was purchased from MP Biomedicals, LLC (Solon, OH, USA) and was reconstituted with distilled water and diluted with saline. Docetaxel was purchased from Sanofi K.K. (Tokyo, Japan) and was diluted with saline just before administration. Paclitaxel was purchased from Wako Pure Chemical Industries, Ltd. (Osaka, Japan) as a fine powder. Paclitaxel was reconstituted with Cremophor EL-ethanol solution (1:1) and diluted tenfold with saline just before administration.

Animals. Female, 5-week-old BALB-nu/nu mice $(\mathrm{CAnN}$. $\mathrm{Cg}-\mathrm{Foxn} 1<\mathrm{nu}>/ \mathrm{CrlCrlj} \mathrm{nu} / \mathrm{nu})$ were obtained from Charles River Laboratories Japan, Inc. (Yokohama, Japan). All animals were allowed to acclimatize and recover from shipping-related stress for 1 week prior to the study. The health of the mice was monitored by daily observation. The animals were allowed free access to chlorinated water and irradiated food, and the animals were kept under a controlled light-dark cycle (12-12 h). All animal experiments were reviewed and approved by the Institutional Animal Care and Use Committee at Chugai Pharmaceutical Co. Ltd.

Cell line and culture conditions. The HER2-positive human breast cancer cell line KPL-4 was kindly provided by Dr. J Kurebayashi (Kawasaki Medical School, Kurashiki, Japan). KPL-4, which is sensitive to trastuzumab in vivo (17) and is estrogen receptor-negative (18), was maintained in Dulbecco's modified Eagle's medium (D-MEM, 1 g/l glucose; Sigma-Aldrich Co. LLC., St. Louis, MO, USA) supplemented with $5 \% \mathrm{FBS}$ at $37^{\circ} \mathrm{C}$ under $5 \% \mathrm{CO}_{2}$.

In vivo tumor growth inhibition studies. Each mouse was inoculated subcutaneously into the second mammary fat pad with $5 \times 10^{6}$ cells/mouse of KPL- 4 . When tumor volumes reached approximately 0.2 to $0.3 \mathrm{~cm}^{3}$, the mice were randomly allocated to control and treatment groups, and treatment with the antitumor agents was started (Day 1). Docetaxel at $10 \mathrm{mg} / \mathrm{kg}$ or vehicle was administered intravenously on the first day of treatment. Paclitaxel was administered at $15 \mathrm{mg} / \mathrm{kg}$ intravenously once a week for 3 weeks. Trastuzumab at $10 \mathrm{mg} / \mathrm{kg}$, pertuzumab at $20 \mathrm{mg} / \mathrm{kg}$, or HuIgG were administered intraperitoneally once a week for 3 weeks. In a separate experiment, trastuzumab was administered at $30 \mathrm{mg} / \mathrm{kg}$. To evaluate the antitumor activity and tolerability of the test agents, tumor volume and body weight were measured twice a week. The tumor volume (TV) was estimated from the equation $V=a b^{2} / 2$, where $a$ and $b$ are tumor length and width, respectively. The percentage of tumor growth inhibition (TGI\%) was calculated as follows: TGI\% $=[1-(\mathrm{TV}$ of treatment group on evaluation day - TV of treatment group on Day 1) / (TV of control group at evaluation day - TV of control group on Day 1)] x 100. Tumor growth rate was calculated as follows: tumor growth rate $=(\mathrm{TV}$ on evaluation day) $/$ (tumor volume on Day 1).

Hematoxylin-eosin staining. Hematoxylin-eosin staining was used for assessment of mitotic tumor cells and mononuclear cells. KPL-4 tumor xenograft tissues were collected 4 days after the initiation of treatment. The tissues were fixed with $10 \%$ neutral buffered formalin and embedded in paraffin. Slide specimens were prepared by sectioning the tissue and staining with hematoxylin-eosin stain. Then, the number of mitotic tumor cells in every 1,000 cells was counted under a microscope. Mononuclear cells infiltrating into tumor tissues were scored as-or 0 , no change; \pm or 1 , very slight; + or 2 , slight; ++ or 3 , moderate; or +++ or 4 , marked.

Terminal deoxynucleotidyl transferase (TdT)-mediated dUTP nick end labeling (TUNEL) assay. Apoptotic cells were assessed by TUNEL assay. KPL-4 tumor xenograft tissues were collected 4 days after the initiation of treatment. The tissues were fixed with $10 \%$ neutral buffered formalin and embedded in paraffin. TUNEL assay was performed and the number of apoptotic cells in every 1,000 tumor cells was counted by Sapporo General Pathology Laboratory Co., Ltd. (Sapporo, Japan).

Ki-67 staining. Proliferating cells were assessed with Ki-67 staining. KPL-4 tumor xenograft tissues were collected 4 days after the initiation of treatment. The tissues were fixed with $10 \%$ neutral buffered formalin and embedded in paraffin. Ki-67 staining was performed and the number of Ki-67-positive cells in every 1,000 tumor cells was counted by Sapporo General Pathology Laboratory Co., Ltd.

Western blotting. KPL-4 tumor xenograft tumors were collected 4 days after the initiation of treatment and immediately frozen in liquid nitrogen and stored at $-80^{\circ} \mathrm{C}$. Tumor samples were homogenized with Cell Lysis Buffer (Cell Signaling Technology, Inc., Beverly, MA, USA) including $10 \mathrm{mM} \mathrm{NaF}, 1 \mu \mathrm{g} / \mathrm{ml}$ aprotinin, and $1 \mathrm{mM}$ phenylmethylsulfonyl fluoride (PMSF). After centrifugation, the resultant supernatant was used for the assays. The lysate was separated on sodium dodecyl sulfate-polyacrylamide gel electrophoresis (SDS-PAGE) gel and transferred onto polyvinylidene fluoride (PVDF) membrane. The membrane was primarily treated with antibodies against p-EGFR, EGFR, p-HER2, HER2, p-HER3, p-ERK, ERK, p-AKT, AKT (Cell Signaling Technology, Inc.), HER3 (Santa Cruz Biotechnology, Inc., Dallas, TX, USA), and $\beta$-actin (Sigma-Aldrich Co. LLC., St. Louis, MO, USA). These proteins were detected by horseradish peroxidase (HRP)-conjugated secondary antibodies (Santa Cruz Biotechnology, Inc.). For HER3, HRP-conjugated 

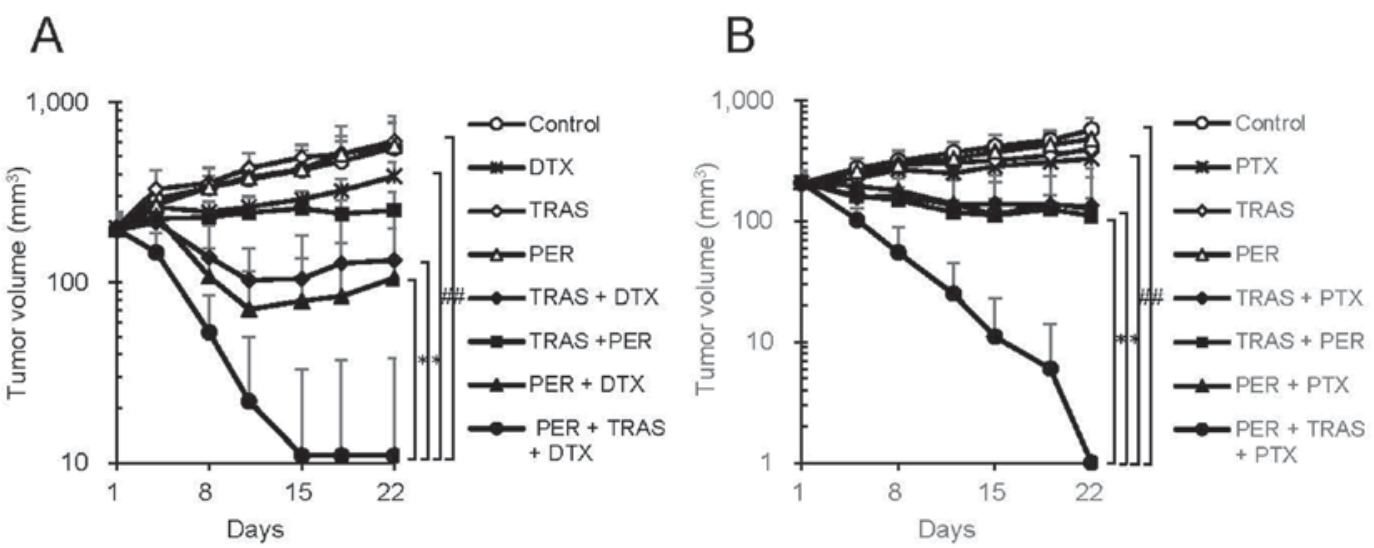

Figure 1. In vivo efficacy of triple-drug combination. (A) Mice bearing KPL-4 tumors were randomly divided into eight groups (n=6 per group) and were treated with trastuzumab, pertuzumab, docetaxel, trastuzumab + docetaxel, trastuzumab + pertuzumab, pertuzumab + docetaxel, or trastuzumab + docetaxel + pertuzumab. As a control, human IgG and vehicle of docetaxel were administered. (B) Mice bearing KPL-4 tumors were randomly divided into eight groups ( $n=6$ per group) and treated with trastuzumab, pertuzumab, paclitaxel, trastuzumab + paclitaxel, trastuzumab + pertuzumab, pertuzumab + paclitaxel, or trastuzumab + paclitaxel + pertuzumab. As a control, human IgG and vehicle of paclitaxel were administered. Data points are mean + standard deviation of the tumor volume $\left(\mathrm{mm}^{3}\right)$. Statistically significant differences are shown as ${ }^{\#} \mathrm{P}<0.05$ and ${ }^{*} \mathrm{P}<0.025$. TRAS, trastuzumab; PER, pertuzumab; DTX, docetaxel; PTX, paclitaxel.

anti-rabbit IgG (Cell Signaling Technology, Inc.) was used as the secondary antibody.

Flow cytometry analysis. To examine the cell cycle of KPL-4 cells in vivo, KPL-4 tumor xenograft tumors were collected 4 days after the initiation of treatment and dissociated with a Tumor Dissociation kit, Human (Miltenyi Biotec GmbH, Bergisch Gladbach, Germany). Then, tumor cells were isolated with a Mouse Cell Depletion kit (Miltenyi Biotec $\mathrm{GmbH}$ ). The cell cycle of the tumor cells was examined by BD Cycletest Plus DNA Reagent kit (BD Biosciences, San Jose, CA, USA). The DNA content in each cell nucleus was determined by FACSVerse (Becton-Dickinson, Franklin Lakes, NJ, USA), and the cell cycle was analyzed by using ModFit LT Version 4 (Verity Software House, Topsham, ME, USA).

Statistical analysis. To analyze the data, Student's t-test was used. For multiple comparisons, significance was determined by hierarchical testing. Firstly, the statistical significance between the control group and the triple-drug combination group (pertuzumab plus trastuzumab plus docetaxel/paclitaxel) was analyzed; secondly, the statistical significance between the docetaxel/paclitaxel group and the triple-drug combination group were analyzed. Finally, Bonferroni correction was applied to establish a threshold for statistical significance between docetaxel/paclitaxel plus trastuzumab group or docetaxel/paclitaxel plus pertuzumab group and the triple-drug combination group. Statistical analyses were conducted using JMP (SAS Institute Japan Ltd., Tokyo, Japan). $\mathrm{P}<0.05$ was considered to indicate a statistically significant difference.

\section{Results}

Establishment of the pertuzumab plus trastuzumab plus docetaxel triple-drug combination treatment model. To examine the internal changes in tumors treated with pertuzumab plus trastuzumab plus docetaxel, we first established a mouse xenograft model by using KPL-4, a HER2-positive human breast cancer cell line, in which the treatment could show sufficient efficacy. In this model, no significant $(\mathrm{P}<0.05)$ anti-tumor effect was observed with docetaxel $(10 \mathrm{mg} / \mathrm{kg})$, pertuzumab $(20 \mathrm{mg} / \mathrm{kg})$ or trastuzumab $(10 \mathrm{mg} / \mathrm{kg})$. However, the triple-drug combination of pertuzumab plus trastuzumab plus docetaxel showed a dramatically stronger antitumor activity compared to either pertuzumab plus docetaxel or trastuzumab plus docetaxel (Fig. 1A). Five out of six mice receiving the triple-drug combination achieved a complete tumor regression 21 days after the treatment started, whereas no mice were cured in either of the double-drug combination groups. The TV, TGI\%, and incidence of tumor-free mice are summarized in Table I. We also examined the efficacy of pertuzumab plus trastuzumab plus paclitaxel as another triple-drug combination. This combination also showed significantly enhanced antitumor activity compared to either pertuzumab plus paclitaxel or trastuzumab plus paclitaxel (Fig. 1B).

In order to eliminate the possibility that the higher antitumor activity of the triple-drug combination was due to the higher overall dosage of anti-HER2 antibodies, the tumor growth rate under the combination of docetaxel plus trastuzumab was compared with the tumor growth rate under the combination of docetaxel plus pertuzumab plus trastuzumab, in which the total dosage of anti-HER2 antibodies in each treatment was equivalent. Tumor regression with the combination of $20 \mathrm{mg} / \mathrm{kg}$ pertuzumab plus $10 \mathrm{mg} / \mathrm{kg}$ trastuzumab plus docetaxel was significantly higher than that with the combination of $30 \mathrm{mg} / \mathrm{kg}$ trastuzumab plus docetaxel (Fig. 2).

Inhibition of HER2 signaling in KPL-4 tumor tissue following treatment with the triple-drug combination. Because trastuzumab and pertuzumab bind to different domains of the HER2 molecule and suppress different aspects of HER2 signaling, i.e. ligand-independent and ligand-induced, trastuzumab 
Table I. Antitumor activity in the KPL-4 HER2-positive breast cancer xenograft model.

\begin{tabular}{lcccc}
\hline & \multicolumn{2}{c}{ Tumor volume $\left(\mathrm{mm}^{3}\right)$} & & Tumor-free mice \\
Treatment & Day 1 & Day 22 & TGI\% on Day 22 & \begin{tabular}{c} 
on 22 \\
\hline Control
\end{tabular} \\
Trastuzumab & $196 \pm 24$ & $560 \pm 206$ & - & $0 / 6$ \\
Pertuzumab & $202 \pm 42$ & $606 \pm 168$ & -11 & $0 / 6$ \\
Docetaxel & $197 \pm 30$ & $574 \pm 262$ & -3 & $0 / 6$ \\
Trastuzumab+docetaxel & $201 \pm 38$ & $389 \pm 76$ & 48 & $0 / 6$ \\
Pertuzumab+docetaxel & $196 \pm 28$ & $133 \pm 102$ & 117 & $0 / 6$ \\
Trastuzumab+pertuzumab & $202 \pm 44$ & $106 \pm 95$ & 126 & $0 / 6$ \\
Pertuzumab+trastuzumab+docetaxel & $196 \pm 25$ & $251 \pm 66$ & 85 & $0 / 6$ \\
\hline
\end{tabular}

Tumor volume was described as mean \pm standard deviation. TGI\%, percentage tumor growth inhibition; Day 1, starting day of treatment; Day 22, 21 days after start of treatment.

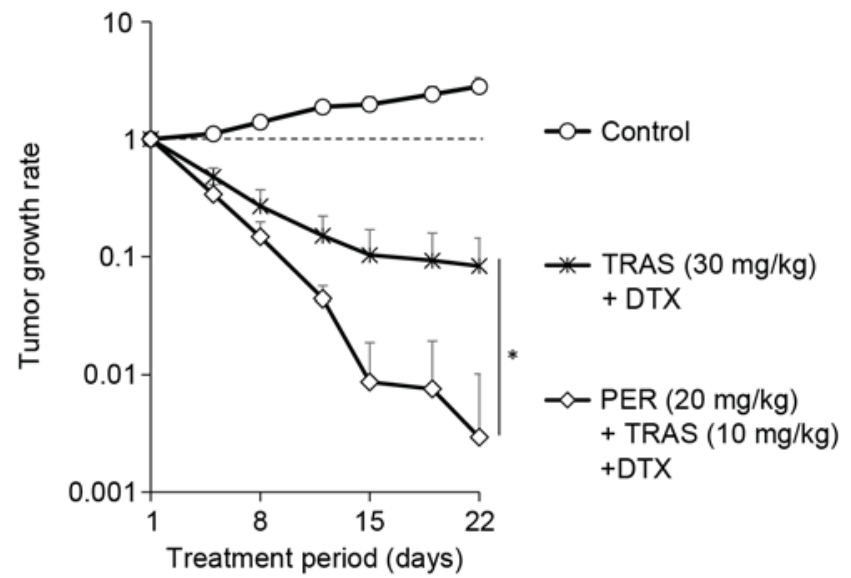

Figure 2. Tumor growth rates under the triple-drug combination and under trastuzumab + docetaxel containing the same amount of antibodies. Mice bearing KPL-4 tumors were randomly divided into three groups ( $\mathrm{n}=6$ per group) and treated with pertuzumab $(20 \mathrm{mg} / \mathrm{kg})+$ trastuzumab $(10 \mathrm{mg} / \mathrm{kg})+$ docetaxel or with trastuzumab $(30 \mathrm{mg} / \mathrm{kg})+$ docetaxel. As a control, human IgG and vehicle of docetaxel were administered. Data points are mean + standard deviation of the tumor growth rate. Statistically significant difference is shown as ${ }^{*} \mathrm{P}<0.05$. TRAS, trastuzumab; PER, pertuzumab; DTX, docetaxel.

and pertuzumab used in combination is expected to inhibit HER2 signaling more effectively. Therefore, we examined HER2 signaling after treatment. Trastuzumab, pertuzumab, or docetaxel alone exhibited little effect on the HER2-related signal transduction. In contrast with the weak suppression by single- or double-drug treatments, the triple-drug combination strongly inhibited the phosphorylation of HER2, EGFR, HER3, ERK, and AKT in tumor tissues (Fig. 3).

Effect of triple-drug combination on apoptosis of tumor cells in vivo. Evaluation of apoptotic cells was performed on tumor tissues obtained 4 days after initiation of treatment. The triple-drug combination significantly enhanced the number of apoptotic cells as compared to the combination of docetaxel plus trastuzumab or docetaxel plus pertuzumab (Fig. 4A and B). We also assessed the number of tumor cells in the mitotic phase,

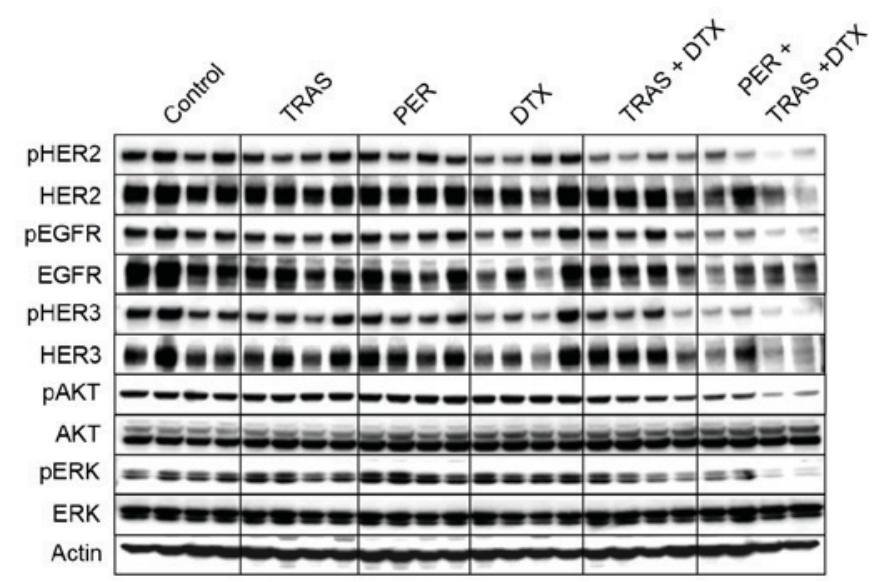

Figure 3. Inhibition of HER2-signal transduction after the triple-drug treatment. Lysates from tumor tissues collected 4 days after starting treatment were used for Western blotting. TRAS, trastuzumab; PER, pertuzumab; DTX, docetaxel ( $n=4$ per group).

because docetaxel was a tubulin depolymerization inhibitor. As shown in Fig. 4C, combination of trastuzumab, pertuzumab, or both of them did not increase the cell number of the mitotic phase induced by docetaxel. These results indicated that trastuzumab and pertuzumab enhanced the induction of apoptosis when combined with docetaxel in spite of the increase of mitotic arrest. In accordance with the apoptosis, proliferating cells assessed by counting the Ki-67 positivity were significantly decreased by the triple-drug combination as compared to the double-drug combinations of docetaxel plus trastuzumab or docetaxel plus pertuzumab (Fig. 4D).

Cell cycle analysis of KPL-4 cells in tumor tissues following treatment with the triple-drug combination. The triple-drug combination might affect the cell cycle in the tumors because it is well known that anti-HER2 antibodies induce G1 arrest and docetaxel induces $M$ arrest in cancer cells treated in vitro (19-22). Therefore, we isolated cancer cells from xenografted tumors treated with trastuzumab, pertuzumab, docetaxel, trastuzumab plus docetaxel, or pertuzumab plus trastuzumab plus docetaxel 
A

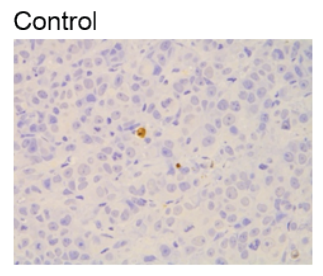

TRAS + DTX

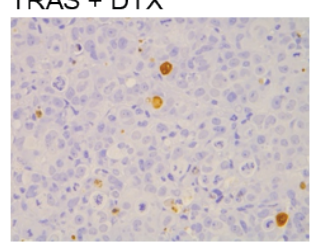

PER

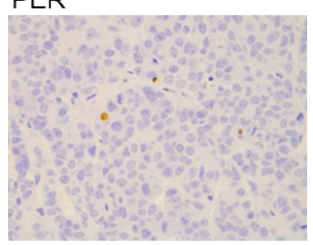

$P E R+T R A S+D T X$

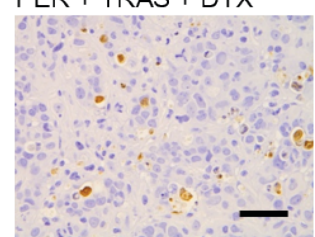

B

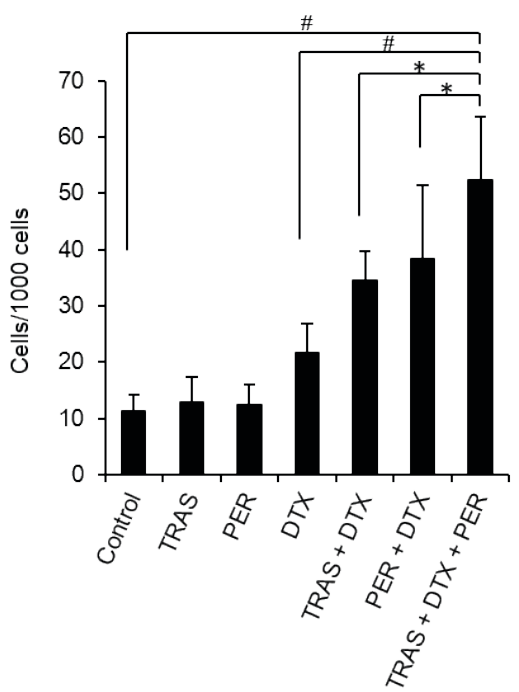

$\mathrm{D}$

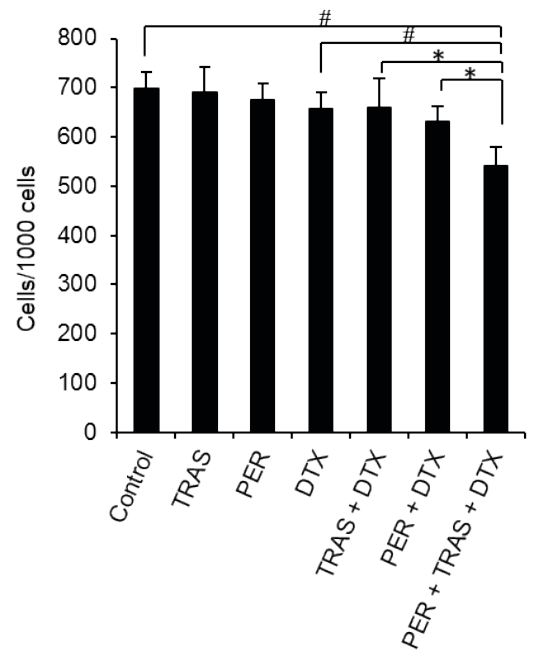

Figure 4. Evaluation of apoptotic and proliferating tumor cells in vivo after the triple-drug treatment. (A) Mice bearing KPL-4 tumors were randomly divided into seven groups ( $\mathrm{n}=6$ or 7 per group) and treated with each combination of agents. Four days after starting treatment, apoptotic tumor cells were histopathologically evaluated. The numbers of (B) apoptotic cells, (C) mitotic cells and (D) Ki-67-positive cells in every 1,000 tumor cells were counted. Scale bar indicated $50 \mu \mathrm{m}$. Statistically significant differences are shown as ${ }^{\#} \mathrm{P}<0.05$ and ${ }^{*} \mathrm{P}<0.025$. TRAS, trastuzumab; PER, pertuzumab; DTX, docetaxel.

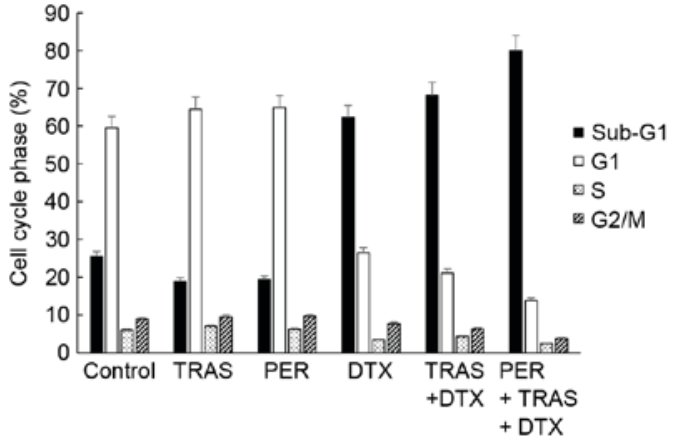

Figure 5. Evaluation of cell cycle of tumor cells in vivo and the percentage of mitotic cells in viable tumor cells after the triple-drug treatment. Tumor tissues were collected 4 days after starting treatment and dissociated. Tumor cells were then isolated and the cell cycle distribution was examined by flow cytometry. The percentage of cells in each cell cycle phase was calculated. TRAS, trastuzumab; PER, pertuzumab; DTX, docetaxel ( $n=3$ per group). and analyzed the cell cycle distribution (Fig. 5). Trastuzumab or pertuzumab treatment did not cause a substantial change in the percentage of $\mathrm{G0} / \mathrm{G1}$ phase cells or other phase cells. Docetaxel treatment did not increase the percentage of $\mathrm{G} 2 / \mathrm{M}$ phase cells, but augmented the percentage of sub-G1 phase cells. In agreement with the number of TUNEL-positive cells (Fig. 4B), treatment with the combination of docetaxel plus pertuzumab plus trastuzumab had a tendency to increase in the percentage of sub-G1 phase cells (74.2\%) as compared to treatment with docetaxel alone or docetaxel plus trastuzumab (62.2 and 60.9\%, respectively). On the other hand, addition of trastuzumab or pertuzumab plus trastuzumab to docetaxel did not affect the G2/M phase population (Fig. 5).

Invasion of mononuclear cells in tumor tissues following treatment with the triple-drug combination of pertuzumab 
A

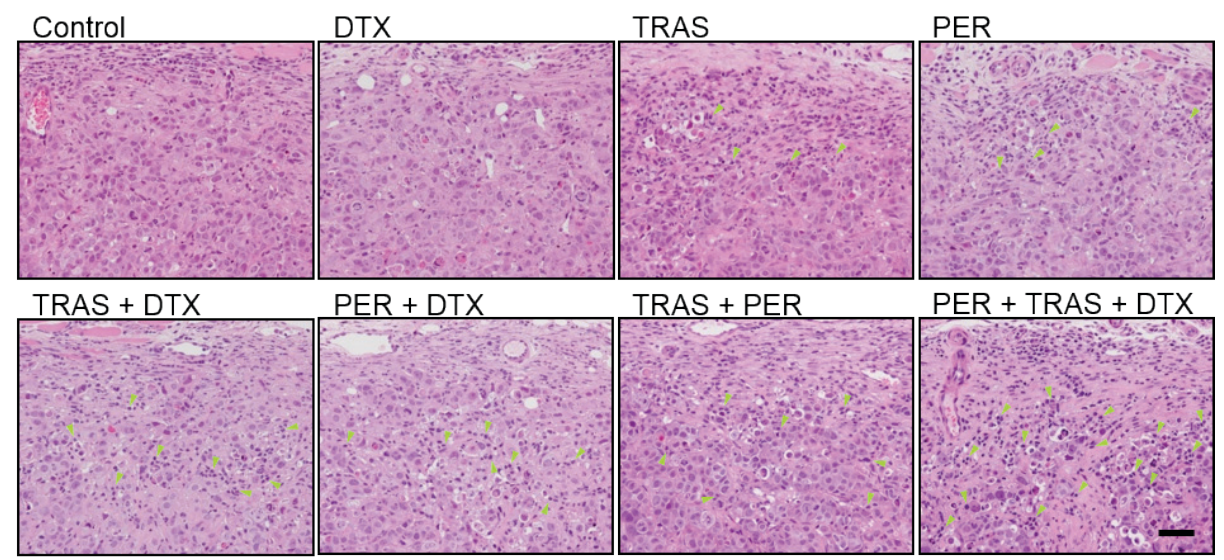

B

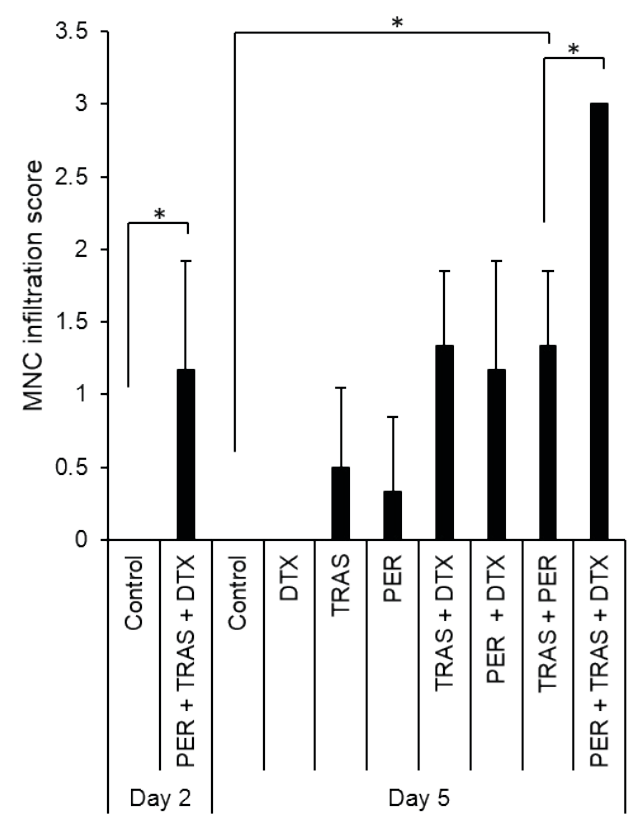

Figure 6. Invasion of MNCs around tumor cells after the triple-drug treatment. (A) Tumor tissues were collected 1 day (Day 2) or 4 days (Day 5) after starting treatment and stained with hematoxylin-eosin stain. Invasion of MNCs were histopathologically evaluated. Arrows show examples of infiltrating MNCs around tumor cells (representative images from Day 5). (B) Infiltration of MNCs was scored as 0: no change; 1: very slight; 2: slight; 3: moderate; or 4: marked, and shown graphically. Scale bar indicated $50 \mu \mathrm{m}$. Statistically significant differences are shown as *P<0.05. TRAS, trastuzumab; PER, pertuzumab; DTX, docetaxel ( $n=6$ or 7 per group).

plus trastuzumab plus docetaxel. To examine innate immune responses, we checked the differences in tumor-infiltrating mononuclear cells (MNCs) in xenografted tumors in nude mice. The invasion of MNCs in the KPL-4 tumor tissues was analyzed 4 days after initiation of treatment (Fig. 6A and B, Table II). Very slight infiltration of MNCs was observed around the tumor cells from xenografted mice treated with trastuzumab or pertuzumab alone, whereas no infiltration was observed around the tumor cells from xenografted mice treated with docetaxel or control. A significant increase in tumor-infiltrating MNCs was observed in the trastuzumab plus pertuzumab group. The MNC infiltration markedly increased with the combination treatment of docetaxel plus trastuzumab plus pertuzumab compared to the trastuzumab plus pertuzumab treatment (Fig. 6A and B). In accordance with the MNC infiltration around the tumor cells, a remarkable increase in single-cell necrosis or apoptosis of the tumor cells and replacement of tumor cell area by connective tissues was observed in the triple-drug combination group (Table II). The MNC infiltration induced by the triple-drug combination was observed as early as the day after the first treatment (Fig. 6B).

\section{Discussion}

To elucidate the mechanism of action of pertuzumab and trastuzumab in combination with docetaxel, in the present study we 


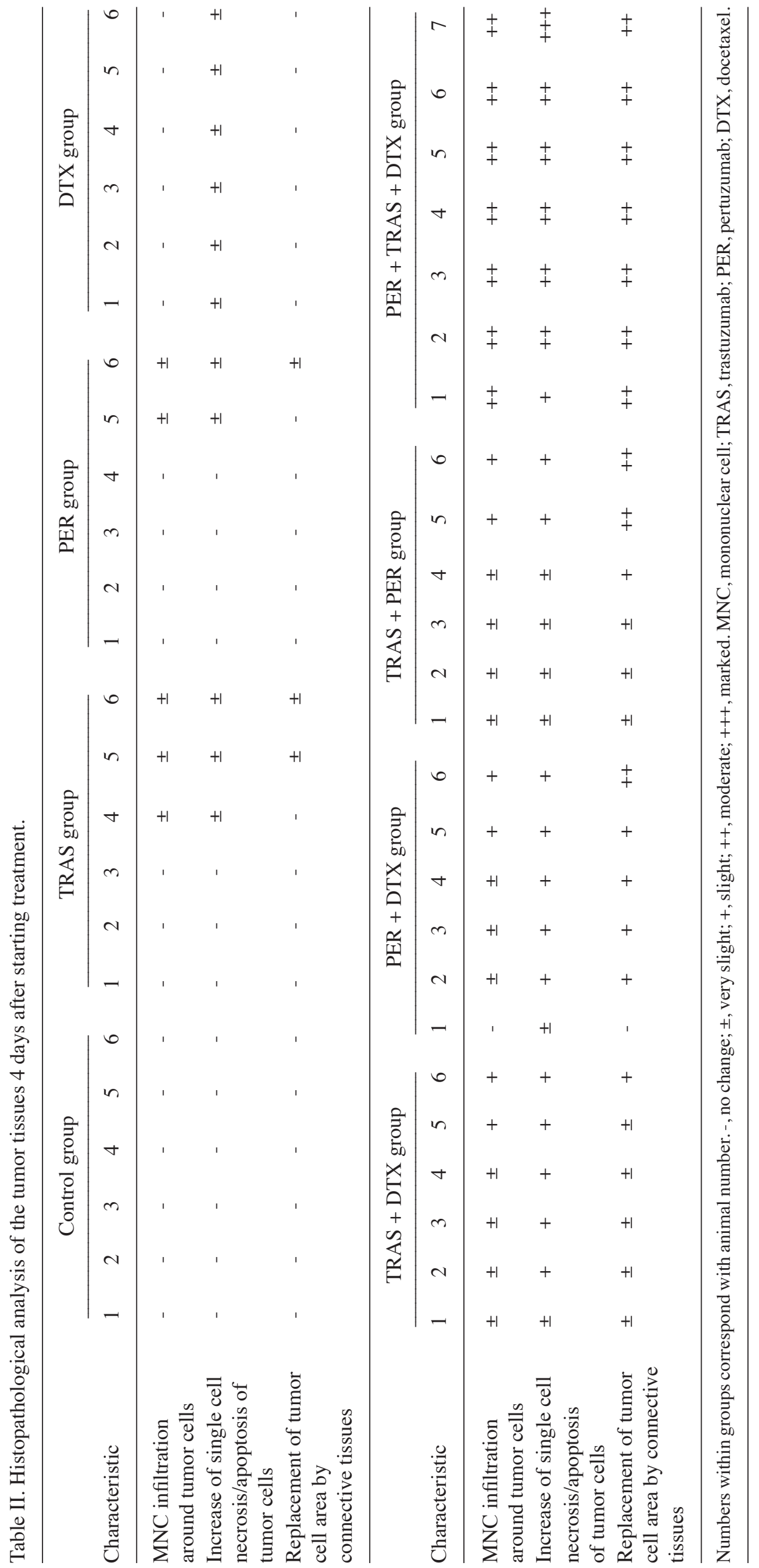


established a mouse xenograft model in which the combination treatments exhibited marked antitumor efficacy even though the dosage of each drug was set to a dosage that had no or weak efficacy on its own (Fig. 1A). The efficacy of the triple-drug combination was significantly higher than the double-drug combinations of trastuzumab plus docetaxel or pertuzumab plus docetaxel (Fig. 1A). Of note, complete tumor regression was observed in five out of six mice during treatment with the triple-drug combination. Similar results were obtained when paclitaxel was used as a combination partner with pertuzumab and trastuzumab (Fig. 1B), indicating that similar combination effects may be obtained with other chemotherapeutic agents besides docetaxel as was reported in the clinical trial (23). In addition, by examining the effect of docetaxel plus trastuzumab in comparison with the effect of docetaxel plus pertuzumab plus trastuzumab when each combination contained an equivalent total dosage of anti-HER2 antibodies, it was shown that the remarkable antitumor effect of the triple-drug combination was not merely due to the higher overall dosage of anti-HER2 antibodies but was due to the synergistic biological effects of pertuzumab, trastuzumab, and docetaxel (Fig. 2). Based on these findings, we used the newly established xenograft model to investigate the mechanisms of action of the triple-drug combination from the aspects of HER 2 signaling inhibition, cell cycle distribution, and infiltration of MNCs into tumor tissues.

Firstly, we analyzed signal transduction relevant to the two antibodies. It was found that the combination of pertuzumab plus trastuzumab plus docetaxel reduced phosphorylation of EGFR, HER3 and their downstream factors AKT and ERK in the tumor tissues more strongly than did either agent alone or the combination of trastuzumab plus docetaxel. The combination of pertuzumab plus trastuzumab has been shown to complementarily suppress HER3-AKT signaling by inhibiting both ligand-induced and ligand-independent HER2-HER3 complex formation (5). It is also reported that suppression of the HER3-AKT pathway activates caspase-3 and induces apoptosis in HER2-positive breast and gastric cancer cell lines $(5,24,25)$ and that EGFR-ERK pathway inhibition induces G1-arrest (26). Consequently, in the present model, apoptotic cells (Fig. 4B) were increased and Ki-67 positive cells (Fig. 4D) were decreased by the triple-drug treatment. These results suggest that the strong antitumor activity and pro-apoptotic activity is at least in part due to enhanced inhibition of HER3-AKT and EGFR-ERK signaling caused by the triple-drug treatment.

Secondly, we analyzed cell cycle distribution of the tumor cells because enhancement of taxane-induced cell cycle arrest could be another mechanism of action. The present study demonstrated that docetaxel, a tubulin depolymerization inhibitor, dramatically increased the number of cells in the sub-G1 phase 4 days after initiation of treatment, and that addition of pertuzumab together with trastuzumab to docetaxel greatly increased numbers of sub-G1 cells and TUNEL-positive cells as compared to numbers following docetaxel or docetaxel plus trastuzumab treatment (Figs. 4A and B, and 5). However, the numbers of cells in the mitotic phase were unchanged between docetaxel and the triple-drug combination groups, suggesting that the triple-drug combination promotes the induction of apoptosis immediately after mitotic arrest.

Thirdly, we analyzed infiltration of MNCs into the tumor tissues because tumor-infiltrating lymphocytes (TILs) have been proved to be a predictive therapeutic marker in early breast cancers (27) as well as in HER2-positive breast cancers (28). Although higher levels of TILs have been shown to be associated with greater trastuzumab benefit in the FinHER trial (29), changes in the number of TILs following trastuzumab treatment as well as changes in the infiltration of MNCs such as NK cells or macrophages into tumor tissues have not yet been clearly analyzed. Here, we demonstrated for the first time that trastuzumab plus pertuzumab enhanced MNC infiltration around the tumor cells 4 days after initiation of treatment. Furthermore, the triple-drug combination dramatically increased the MNC infiltration compared with the trastuzumab plus pertuzumab combination. Of note, the MNC infiltration following the triple-drug combination was observed as early as the day after the first treatment, before tumor growth inhibition had become apparent. These results suggest that the enhanced recruitment of MNCs into tumor tissues contributes to inhibition of tumor growth in the triple-drug combination, possibly through ADCC $(13,14)$. Indeed, it has been shown that the number of KPL-4 cells killed by NK cells in vitro depends on the amount of antibodies (14). In addition, docetaxel has been reported to increase serum IL-2 level and enhance NK cell activity in patients with breast cancer (30). Thus, we consider that the triple-drug combination of trastuzumab plus docetaxel plus pertuzumab could cooperatively enhance ADCC activity and contribute to tumor shrinkage in the KPL-4 xenografted mouse model. Although athymic nude mice do retain NK cells, it has to be admitted that xenograft models using nude mice as hosts are inadequate in regard to the immune system. In order to investigate the mechanism of action of the triple-drug combination more precisely in terms of immune reactions, models appropriate for the evaluation, such as models using humanized mice, should be utilized.

In conclusion, the synergistic efficacy of the triple-drug combination of pertuzumab in combination with trastuzumab and docetaxel against a HER2-positive breast cancer model was considered to be produced by the integration of two mechanisms: the inhibition of HER2 signaling pathways by anti-HER2 antibodies promoted the apoptosis evoked after docetaxel-induced mitotic arrest; docetaxel enhanced the infiltration of tumor tissues by mononuclear cells, and this increased infiltration may have upregulated the antibody-dependent cellular cytotoxicity. This is the first report to reveal the mechanism of action of the superior antitumor effect of the triple-drug combination therapy.

\section{Acknowledgements}

We greatly appreciate Hiromi Sawamura, Masako Miyazaki, and Kumiko Kondo (Product Research department, Chugai) for their excellent technical assistance; and Dr Kaori FujimotoOuchi, Dr Mieko Yanagisawa, and Dr Yasushi Yoshimura (Product Research department, Chugai) for helpful discussion and comments regarding the study.

\section{References}

1. Ravdin PM and Chamness GC: The c-erbB-2 proto-oncogene as a prognostic and predictive marker in breast cancer: A paradigm for the development of other macromolecular markers-a review. Gene 159: 19-27, 1995. 
2. Slamon DJ, Clark GM, Wong SG, Levin WJ, Ullrich A and McGuire WL: Human breast cancer: Correlation of relapse and survival with amplification of the HER-2/neu oncogene. Science 235: 177-182, 1987

3. Dawood S, Broglio K, Buzdar AU, Hortobagyi GN and Giordano SH: Prognosis of women with metastatic breast cancer by HER 2 status and trastuzumab treatment: An institutional-based review. J Clin Oncol 28: 92-98, 2010.

4. Ross JS, Slodkowska EA, Symmans WF, Pusztai L, Ravdin PM and Hortobagyi GN: The HER-2 receptor and breast cancer: Ten years of targeted anti-HER-2 therapy and personalized medicine. Oncologist 14: 320-368, 2009.

5. Junttila TT, Akita RW, Parsons K, Fields C, Lewis Phillips GD, Friedman LS,Sampath D and Sliwkowski MX: Ligand-independent HER2/HER3/PI3K complex is disrupted by trastuzumab and is effectively inhibited by the PI3K inhibitor GDC-0941. Cancer Cell 15: 429-440, 2009.

6. Molina MA, Codony-Servat J, Albanell J, Rojo F, Arribas J and Baselga J: Trastuzumab (Herceptin), a humanized anti-Her2 receptor monoclonal antibody, inhibits basal and activated Her2 ectodomain cleavage in breast cancer cells. Cancer Res 61 4744-4749, 2001.

7. Beano A, Signorino E, Evangelista A, Brusa D, Mistrangelo M, Polimeni MA, Spadi R, Donadio M, Ciuffreda L and Matera L: Correlation between NK function and response to trastuzumab in metastatic breast cancer patients. J Transl Med 6: 25, 2008

8. Barok M, Isola J, Pályi-Krekk Z, Nagy P, Juhász I, Vereb G, Kauraniemi P, Kapanen A, Tanner M, Vereb G and Szöllösi J: Trastuzumab causes antibody-dependent cellular cytotoxicity-mediated growth inhibition of submacroscopic JIMT-1 breast cancer xenografts despite intrinsic drug resistance. Mol Cancer Ther 6: 2065-2072, 2007.

9. Marty M, Cognetti F, Maraninchi D, Snyder R, Mauriac L, Tubiana-Hulin M, Chan S, Grimes D, Antón A, Lluch A, et al: Randomized phase II trial of the efficacy and safety of trastuzumab combined with docetaxel in patients with human epidermal growth factor receptor 2-positive metastatic breast cancer administered as first-line treatment: The M77001 study group. J Clin Oncol 23: 4265-4274, 2005

10. Slamon DJ, Leyland-Jones B, Shak S, Fuchs H, Paton V, Bajamonde A, Fleming T, Eiermann W, Wolter J, Pegram M, et al: Use of chemotherapy plus a monoclonal antibody against HER2 for metastatic breast cancer that overexpresses HER2. N Engl J Med 344: 783-792, 2001.

11. Franklin MC, Carey KD, Vajdos FF, Leahy DJ, de Vos AM and Sliwkowski MX: Insights into ErbB signaling from the structure of the ErbB2-pertuzumab complex. Cancer Cell 5: 317-328, 2004.

12. Agus DB, Akita RW, Fox WD, Lewis GD, Higgins B, Pisacane PI, Lofgren JA, Tindell C, Evans DP, Maiese K, et al: Targeting ligand-activated ErbB2 signaling inhibits breast and prostate tumor growth. Cancer Cell 2: 127-137, 2002.

13. Yamashita-Kashima Y, Iijima S, Yorozu K, Furugaki K, Kurasawa M, Ohta M and Fujimoto-Ouchi K: Pertuzumab in combination with trastuzumab shows significantly enhanced antitumor activity in HER2-positive human gastric cancer xenograft models. Clin Cancer Res 17: 5060-5070, 2011.

14. Scheuer W, Friess T, Burtscher H, Bossenmaier B, Endl J and Hasmann M: Strongly enhanced antitumor activity of trastuzumab and pertuzumab combination treatment on HER2-positive human xenograft tumor models. Cancer Res 69 : 9330-9336, 2009

15. Baselga J, Cortés J, Kim SB, Im SA, Hegg R, Im YH, Roman L, Pedrini JL, Pienkowski T, Knott A, et al: Pertuzumab plus trastuzumab plus docetaxel for metastatic breast cancer. N Engl J Med 366: 109-119, 2012.
16. Swain SM, Kim SB, Cortés J, Ro J, Semiglazov V, Campone M, Ciruelos E, Ferrero JM, Schneeweiss A, Knott A, et al: Pertuzumab, trastuzumab, and docetaxel for HER 2-positive metastatic breast cancer (CLEOPATRA study): Overall survival results from a randomised, double-blind, placebo-controlled, phase 3 study. Lancet Oncol 14: 461-471, 2013.

17. Kurebayashi J, Otsuki T, Tang CK, Kurosumi M, Yamamoto S, Tanaka K, Mochizuki M, Nakamura $\mathrm{H}$ and Sonoo H.: Isolation and characterization of a new human breast cancer cell line, KPL-4, expressing the Erb B family receptors and interleukin-6. Br J Cancer 79: 707-717, 1999.

18. Kunisue H, Kurebayashi J, Otsuki T, Tang CK, Kurosumi M, Yamamoto S, Tanaka K, Doihara H, Shimizu N and Sonoo H: Anti-HER2 antibody enhances the growth inhibitory effect of anti-oestrogen on breast cancer cells expressing both oestrogen receptors and HER2. Br J Cancer 82: 46-51, 2000.

19. Le XF, McWatters A, Wiener J, Wu JY, Mills GB and Bast RC Jr: Anti-HER2 antibody and heregulin suppress growth of HER2-overexpressing human breast cancer cells through different mechanisms. Clin Cancer Res 6: 260-270, 2000.

20. Lane HA, Beuvink I, Motoyama AB, Daly JM, Neve RM and Hynes NE: ErbB2 potentiates breast tumor proliferation through modulation of p27Kip1-Cdk2 complex formation: Receptor overexpression does not determine growth dependency. Mol Cell Biol 20: 3210-3223, 2000

21. Yakes FM, Chinratanalab W, Ritter CA, King W, Seelig S and Arteaga CL: Herceptin-induced inhibition of phosphatidylinositol-3 kinase and Akt is required for antibody-mediated effects on p27, cyclin D1, and antitumor action. Cancer Res 62: 4132-4141, 2002.

22. Ringel I and Horwitz SB: Studies with RP 56976 (taxotere): A semisynthetic analogue of taxol. J Natl Cancer Inst 83: 288-291, 1991.

23. Dang C, Iyengar N, Datko F, D'Andrea G, Theodoulou M, Dickler M, Goldfarb S, Lake D, Fasano J, Fornier M, et al: Phase II study of paclitaxel given once per week along with trastuzumab and pertuzumab in patients with human epidermal growth factor receptor 2-positive metastatic breast cancer. J Clin Oncol 33: 442-447, 2015.

24. Piechocki MP, Yoo GH, Dibbley SK and Lonardo F: Breast cancer expressing the activated HER2/neu is sensitive to gefitinib in vitro and in vivo and acquires resistance through a novel point mutation in the HER2/neu. Cancer Res 67: 6825-6843, 2007.

25. Yamashita-Kashima Y, Shu S, Harada N and Fujimoto-Ouchi K: Enhanced antitumor activity of trastuzumab emtansine (T-DM1) in combination with pertuzumab in a HER2-positive gastric cancer model. Oncol Rep 30: 1087-1093, 2013.

26. Huang Y, Yu T, Fu X, Chen J, Liu Y, Li C, Xia Y, Zhang Z and Li L: EGFR inhibition prevents in vitro tumor growth of salivary adenoid cystic carcinoma. BMC Cell Biol 14: 13, 2013.

27. Melichar B, Študentova H, Kalábová H, Vitásková D, Čermáková $\mathrm{P}$, Hornychová H and Ryška A: Predictive and prognostic significance of tumor-infiltrating lymphocytes in patients with breast cancer treated with neoadjuvant systemic therapy. Anticancer Res 34: 1115-1125, 2014.

28. Zardavas D, Fouad TM and Piccart M: Optimal adjuvant treatment for patients with HER2-positive breast cancer in 2015. Breast 24 (Suppl 2): S143-S148, 2015.

29. Loi S, Michiels S, Salgado R, Sirtaine N, Jose V, Fumagalli D, Kellokumpu-Lehtinen PL, Bono P, Kataja V, Desmedt C, et al: Tumor infiltrating lymphocytes are prognostic in triple negative breast cancer and predictive for trastuzumab benefit in early breast cancer: Results from the FinHER trial. Ann Oncol 25: 1544-1550, 2014.

30. Tsavaris N, Kosmas C, Vadiaka M, Kanelopoulos P and Boulamatsis D: Immune changes in patients with advanced breast cancer undergoing chemotherapy with taxanes. Br J Cancer 87: $21-27,2002$ 